\title{
Provider Response to Critical Action Values for Hypoglycemia in the Ambulatory Setting: a Retrospective Cohort Study
}

\author{
Aditya Ashok, MD, MSc' , Mohammed S. Abusamaan, MD, MPH², \\ Penelope Parker, $M H S^{2}$, Scott J. Pilla, MD, MHS', and Nestoras N. Mathioudakis, \\ $M D, M H S^{2}$ (D) \\ 'Division of General Internal Medicine, Johns Hopkins University School of Medicine Baltimore, MD, USA; ${ }^{2}$ Division of Endocrinology, Diabetes, \& \\ Metabolism, Johns Hopkins University School of Medicine Baltimore, MD, USA.
}

BACKGROUND: The blood glucose level triggering a critical action value (CAV) for hypoglycemia is not standardized, and associated outcomes are unknown.

OBJECTIVE: To evaluate the clinical consequences of, and provider responses to, CAVs for hypoglycemia.

DESIGN: Retrospective cohort study at Johns Hopkins Hospital and Johns Hopkins Bayview Medical Center between April 1, 2013, and January 31, 2017.

PARTICIPANTS: Patients with an ambulatory serum glucose $<50 \mathrm{mg} / \mathrm{dL}$. Point-of-care capillary glucose and whole blood glucose samples were excluded.

MAIN MEASURES: Electronic medical record (EMR) review for providers' documented response to CAV, associated patient symptoms, and serious adverse events.

KEY RESULTS: We analyzed $209 \mathrm{CAVs}$ for hypoglycemia from 154 patients. The median age (IQR) was 59 years (46, 69), 89 (57.8\%) were male, and 96 (62.3\%) were black. Provider-to-patient contact occurred in 128 of 209 $(61.2 \%)$ episodes, among which no documented etiology was observed for 81 of 128 (63.3\%), no recommendations were provided in 32 of 128 (25.0\%), and no patientreported hypoglycemic symptoms were documented in 103 of 128 (80.5\%). Serious adverse events were documented in 4 of 128 episodes (3.1\%), two required glucagon administration, and three required an ED visit. Providerto-patient contact was associated with the patient having malignant neoplasm (adjusted OR 3.63, $p=0.045$ ) or a hypoglycemic disorder (adjusted OR 7.70, $p=0.018$ ) and inversely associated with a longer time from specimen collection to EMR result (adjusted OR 0.90 per hour, $p=$ 0.016).

CONCLUSIONS: There is inconsistent provider-to-patient contact following CAVs for hypoglycemia, and the etiology and symptoms of hypoglycemia were infrequently documented. There were few serious documented adverse events associated with hypoglycemia, although undocumented events may have occurred, and the incidence of serious adverse events in non-contacted patients remains unknown. These findings demonstrate a need to standardize provider response to CAVs for hypoglycemia. Decreasing the lag time between sample collection and

Received May 9, 2020

Accepted September 7, 2020

Published online September 15, 2020 laboratory result reporting may increase provider-topatient contact.

KEY WORDS: Hypoglycemia; Critical action value; Ambulatory; Glucose.

J Gen Intern Med 36(5):1244-9

DOI: $10.1007 / \mathrm{s} 11606-020-06225-\mathrm{y}$

(C) Society of General Internal Medicine 2020

\section{INTRODUCTION}

A critical action value (CAV) is a test result that is potentially life threatening if not promptly intervened upon. ${ }^{1}$ Hypoglycemia is one such example as, if left untreated, it can lead to altered mentation, coma, or death. ${ }^{2}$ The International Hypoglycaemia Study Group considers a glucose concentration < $54 \mathrm{mg} / \mathrm{dl}(3.0 \mathrm{mmol} / \mathrm{L})$ to be clinically important, serious hypoglycemia. ${ }^{3}$ Translating these concepts into a CAV for hypoglycemia that can be applied widely to an ambulatory population is not straightforward. One of the challenges in defining a CAV threshold for hypoglycemia may be the difficulty in ascribing risk in relation to a given blood glucose (BG) value for those with and without diabetes. Factors that have been shown to impact rates of severe hypoglycemia include type of diabetes and duration of insulin use. ${ }^{46}$

There is variability regarding the definition of CAV for hypoglycemia among major medical centers in the USA. At our institution, BG cutoffs of $<60 \mathrm{mg} / \mathrm{dL}$ and $<50 \mathrm{mg} / \mathrm{dL}$ are used as CAV BGs for inpatients/emergency and general ambulatory patients, respectively. By comparison, the Massachusetts General Hospital and Mayo Clinic use plasma glucose cutoffs of $<40 \mathrm{mg} /$ $\mathrm{dL}$ and $\leq 50 \mathrm{mg} / \mathrm{dL}$, respectively, in all patient settings. ${ }^{7,} 8$

Identifying a critical action BG value in the general population is inherently challenging, but knowing what to do in response to CAVs for hypoglycemia presents even further obstacles. The time between BG value resulting in the electronic medical record (EMR), laboratory notification of the ordering provider, and provider's ability to contact the patient all represent potential delays to action and sources of practice variation. To our knowledge, no studies have been conducted that have examined provider responses to CAVs for 
hypoglycemia in the outpatient setting. We sought to address this gap by characterizing outpatient CAVs for hypoglycemia and provider responses to it at our institutions.

\section{METHODS}

\section{Patient Population and Design}

We performed a retrospective cohort study using data from the EMR of Johns Hopkins Hospital (JHH) and Johns Hopkins Bayview Medical Center (JHBMC), both tertiary care academic medical centers in Baltimore, MD. Patients were included if they had an ambulatory serum glucose measurement at our clinical laboratories between April 1, 2013, and January 31, 2017, and had one or more serum glucose $<50 \mathrm{mg} / \mathrm{dL}$. Point-of-care capillary glucose and whole blood glucose samples were excluded. No laboratory specimens collected outside of JHH and JHBMC (e.g., commercial laboratories) were included in the study. Patients whose medical records were designated as "restricted access" in the EMR (e.g., confidentiality for psychiatric reasons) were excluded. This study received approval from the Johns Hopkins Institutional Review Board.

The glucose threshold of $<50 \mathrm{mg} / \mathrm{dL}$ was selected because it is the current CAV threshold in the ambulatory setting at both of our hospitals. Details regarding our laboratory method of glucose analysis have been previously described. ${ }^{9}$ According to our institution's CAV policy, a BG $<50 \mathrm{mg} / \mathrm{dL}$ will trigger an immediate page to the ordering provider; if the ordering provider fails to respond after several attempts, the on-call pathology resident is contacted.

\section{Exposures}

Demographics, diabetes type, glucose-lowering medications, and conditions associated with hypoglycemia were abstracted from the patient's problem list, medical history, or patient encounter by clinical data analysts using the relevant International Classification of Diseases (ICD)-10 codes as previously described. ${ }^{9}$ Predictor variables were selected based on review of the clinical literature to identify established risk factors for hypoglycemia and inclusion of systems factors that could be related to hypoglycemia. For patients with diabetes, the primary driver of hypoglycemia risk is the specific antihyperglycemic medication used, with increasing risk expected with progression from low-risk non-insulin antihyperglycemic medications (e.g., metformin) to oral insulin secretagogues to insulin. For patients taking more than one antihyperglycemic medication, the highest risk medication was used to categorize the patient. Hypoglycemic disorder was defined as having an ICD-10 code for hypoglycemia (E16.2) or insulinoma (D13.7, E16.1).

For each CAV BG, we collected information about specimen collection time, EMR result time, and time of provider-to-patient contact through manual chart review. Notably, the time from BG resulting in the EMR to notification of the provider by the core laboratory could not be systematically captured in this study owing to inconsistent documentation by the core laboratory.

\section{Outcomes}

The primary outcomes of interest were provider and patient response to CAVs for hypoglycemia, patient-reported hypoglycemic symptoms, and hypoglycemic-related serious adverse events. Provider contact was considered present if there was any documentation by the notified provider of communication with the patient or patient representative regarding the CAV for hypoglycemia using any method (phone, electronic, etc.). If the provider explicitly stated the date/time he/she spoke with the patient or patient representative, this time was captured as the patient contact time. In the event that the provider did not explicitly document the date/time the communication actually occurred, the contact time was considered to be the date/time of completion (signing) of any method of documentation that included mention of the CAV BG. Provider documentation of any presumed etiology of hypoglycemia and their recommendations for patient action were identified using pre-specified categories. Patient-reported symptoms of hypoglycemia were collected from providers' documentation. Hypoglycemia-related serious adverse events were defined as any of the following: need for EMS or paramedic support, need for glucagon, need for intravenous dextrose, loss of consciousness or syncope, hypoglycemiarelated $\mathrm{ED}$ visit (to any hospital within our health system) within $24 \mathrm{~h}$ of CAV for hypoglycemia, hypoglycemia-related motor vehicle accident, seizure, cardiac ischemia or arrhythmia, coma, or death.

\section{Statistical Analysis}

Descriptive statistics were used to describe the study population and outcomes. All continuous variables were non-normally distributed, so were presented as medians and interquartile ranges. We used logistic regression to determine the association between provider-to-patient contact in response to a CAV event (outcome) and characteristics of patients and the hypoglycemic episodes (predictors). To account for non-independence of the outcome for patients with repeated episodes, robust standard errors were determined using clustering analysis per unique patients. ${ }^{10,11}$ Our unadjusted model explored the association between provider-to-patient contact as a binary-dependent variable with each of the independent predictor variables. A fully adjusted model explored the association between this outcome with each of the predictor variables, after adjustment for all other variables. Statistical analysis was conducted using Stata 15.1 (StataCorp. 2017. Stata Statistical Software: Release 15. College Station, $\mathrm{TX}) . p<0.05$ was considered statistically significant.

\section{RESULTS}

There were 213 CAVs for hypoglycemic episodes identified in the ambulatory setting during the study period. After 
exclusion of four episodes due to inability to access the patients' EMR, 209 CAVs for hypoglycemic episodes representing 154 unique patients were included in the analysis. Among these patients, $124(81.0 \%)$ had a single CAV hypoglycemic episode, $18(11.7 \%)$ had two episodes, 8 (5.2\%) had three episodes, and $4(2.6 \%)$ had 4 or more episodes. The range of episodes per patient was 1 to 11 .

Table 1 shows the characteristics of the study population at the time of the first hypoglycemic CAV episode. Overall, the median (IQR) age was $59(46,49)$ years, and there was a slight male predominance $(57.8 \%)$. The majority of patients were black $(62.3 \%)$ and had type 2 diabetes mellitus (T2DM) (63.6\%). After T2DM, the most common hypoglycemicrelated comorbidities were congestive heart failure (CHF) (24.0\%), HIV (22.1\%), chronic liver diseases or hepatic failure (9.7\%), and end stage renal diseases (7.1\%). Among the 154 patients with CAV hypoglycemia, 65 (42.2\%) used insulin and $9(5.8 \%)$ used oral insulin secretagogues (with or without other antihyperglycemic agents). The median (IQR) hypoglycemic BG value was $44(38,47) \mathrm{mg} / \mathrm{dL}$, with a median time from $\mathrm{BG}$ specimen collection to electronic reporting of the laboratory result of $2.9(1.4,5.0)$ hours.

Table 1 Patient Characteristics at Time of First Episode of CAV for Hypoglycemia.

\begin{tabular}{|c|c|}
\hline Factor & $\begin{array}{l}N=154 \\
\text { patients }^{\mathrm{a}}\end{array}$ \\
\hline $\begin{array}{l}\text { Hypoglycemic BG measurement, mg/dL, median } \\
\text { (IQR) }\end{array}$ & $44(38,47)$ \\
\hline $\begin{array}{l}\text { Time (hours) from specimen collection to blood } \\
\text { glucose result, median (IQR) }\end{array}$ & $2.9(1.4,5.0)$ \\
\hline $\begin{array}{l}\text { Range (hours) from specimen collection to blood } \\
\text { glucose result }\end{array}$ & $0.2-25.2$ \\
\hline Age (year), median (IQR) & $59(46,69)$ \\
\hline Sex: Male, no. $(\%)$ & $89(57.8)$ \\
\hline \multicolumn{2}{|l|}{ Race, no. $(\%)$} \\
\hline Non-Black & $58(37.7)$ \\
\hline Black & $96(62.3)$ \\
\hline \multicolumn{2}{|l|}{ Hypoglycemic risk factors, no. (\%) } \\
\hline Type 1 diabetes mellitus & $10(6.5)$ \\
\hline Type 2 diabetes mellitus & $98(63.6)$ \\
\hline Hypoglycemic disorder & $9(5.8)$ \\
\hline Adrenal insufficiency & $1(0.6)$ \\
\hline Malnutrition and eating disorders & $8(5.2)$ \\
\hline Post-bariatric or GI surgery & $1(0.6)$ \\
\hline Congestive heart failure & $37(24.0)$ \\
\hline Acute kidney injury & $1(0.6)$ \\
\hline End stage renal diseases & $11(7.1)$ \\
\hline Chronic liver diseases or hepatic failure & $15(9.7)$ \\
\hline HIV & $34(22.1)$ \\
\hline Malignant neoplasm & $10(6.5)$ \\
\hline \multicolumn{2}{|l|}{ Antihyperglycemic medications } \\
\hline None & $79(51.3)$ \\
\hline $\begin{array}{l}\text { Low-risk antihyperglycemics }{ }^{\mathrm{b}} \text { (no insulin, no } \\
\text { secretagogues) }\end{array}$ & $1(0.6)$ \\
\hline $\begin{array}{l}\text { Insulin secretagogues }{ }^{\mathrm{c}}( \pm \text { low-risk antihyperglyce- } \\
\text { mics, no insulin) }\end{array}$ & $9(5.8)$ \\
\hline Insulin ( \pm any other antihyperglycemics) & $65(42.2)$ \\
\hline
\end{tabular}

${ }^{a}$ There were a total of 209 hypoglycemic episodes, but results are summarized for first CAV episode per patient $(N=154)$ over the study period

${ }^{b}$ Metformin, dipeptidyl-peptidase-4 inhibitors, glucagon-like 1 receptor agonists, thiazolidinediones, sodium-glucose cotransporter-2 inhibitor, and alpha glucosidase inhibitors

${ }^{c}$ Sulfonylureas and meglitinides
Table 2 Provider and Patient Response to Hypoglycemic CAV.

\begin{tabular}{ll}
\hline \hline & No. (\%) \\
\hline Documentation of patient contact by provider & $128 / 209$ \\
& $(61.2)$ \\
Time from CAV BG result to documented provider-to- & $1.5(0.5$, \\
patient contact in hours, median (IQR)* & $4.7)$ \\
Time from CAV BG specimen collection to documented & $4.5(2.6$, \\
provider-to-patient contact in hours, median (IQR)* & $8.6)$ \\
Documented etiologies of hypoglycemia (if contacted) & \\
None & $81 / 128$ \\
& $(63.3)$ \\
Prolonged fasting or reduce nutritional intake & $34 / 128$ \\
& $(26.6)$ \\
Dose or type of antihyperglycemic medication & $4 / 128(3.1)$ \\
Patient took antihyperglycemic medication incorrectly & $4 / 128(3.1)$ \\
Suspected spurious laboratory value & $3 / 128(2.3)$ \\
Dumping syndrome & $1 / 128(0.8)$ \\
Steroid taper & $1 / 128(0.8)$ \\
Documented recommendation(s) regarding patient action & $($ if contacted) \\
None & $32 / 128$ \\
& $(25.0)$ \\
Address with primary care physician, endocrinologist, & $25 / 128$ \\
or ordering provider & $(19.5)$ \\
Eat a meal or consume carbohydrates immediately & $12 / 128(9.4)$ \\
Continue to eat regularly, consume carbohydrates if & $12 / 128(9.4)$ \\
symptomatic, or modify diet & \\
Call 911 or go to ED if symptoms recur or persist & $12 / 128(9.4)$ \\
Stop or temporarily hold antihyperglycemic & $9 / 128(7.0)$ \\
medication(s) & \\
Reduce or modify dose of antihyperglycemic & $8 / 128(6.3)$ \\
medication(s) & $7 / 128(5.5)$ \\
Increased vigilance of blood glucose monitoring & $6 / 128(3.9)$ \\
Keep candy or glucose tablets on hand at all times & $3 / 128(2.3)$ \\
Repeat blood glucose test & $2 / 128(1.6)$ \\
Visit ED or urgent care center immediately & $2 / 128(1.6)$ \\
Have third party present to observe patient for period of & \\
time & $1 / 128(0.8)$ \\
Ensure that glucagon emergency kit is available and not & \\
expired & $1 / 128(0.8)$ \\
Call for questions, concerns, or advice & \\
\hline & \\
&
\end{tabular}

$* N=128$ episodes where provider-to-patient contact occurred

Table 2 summarizes the provider and patient response to hypoglycemic CAVs. There was documentation that the patient was contacted regarding the hypoglycemic result in $61.2 \%$ of hypoglycemic episodes. Among the 128 episodes in which the provider documented communication with the patient, the median (IQR) time from the resulting of the hypoglycemic value in the EMR to documentation of patient contact was $1.5(0.5,4.7)$ hours. The total median time between specimen collection and patient/provider communication was $4.5(2.6,8.6)$ hours. Among the 128 episodes in which the patient was contacted by the provider, most commonly no hypoglycemic etiology was documented (63.3\%). The most common etiology identified was prolonged fasting or reduced nutritional intake $(26.6 \%)$, followed by dose or type of antihyperglycemic medication (3.1\%), and patient incorrectly taking antihyperglycemic medication (3.1\%). The provider suspected a spurious laboratory result in $2.3 \%$ of episodes. A documented clinical recommendation by the provider in response to the hypoglycemic CAV was identified in 96 of these 128 episodes (75\%). The most common recommendations were to address the laboratory abnormality with another provider, consume carbohydrates, go to ED if symptoms worsen, or adjust antihyperglycemic medications. 
Table 3 Association of Hypoglycemic Episode Characteristics and Documented Provider-to-Patient Contact.

\begin{tabular}{lll}
\hline \hline & $\begin{array}{l}\text { Unadjusted OR } \\
(\mathbf{9 5 \%} \mathbf{C I})\end{array}$ & $\begin{array}{l}\text { Adjusted } \mathbf{O R}^{\mathbf{a}} \\
\mathbf{( 9 5 \%} \mathbf{C I})\end{array}$ \\
\hline $\begin{array}{l}\text { Hypoglycemic BG value, } \\
\text { mg/dL }\end{array}$ & $0.96(0.92,1.00)$ & $0.96(0.91,1.00)$ \\
$\begin{array}{l}\text { Time from BG specimen } \\
\text { collection to result in }\end{array}$ & $0.91(0.84,0.99)$ & $0.90(0.83,0.98)$ \\
$\begin{array}{l}\text { hours } \\
\text { Age (year) }\end{array}$ & $1.01(0.99,1.03)$ & $1.00(0.98,1.02)$ \\
Male (reference: Female) & $0.69(0.37,1.27)$ & $0.92(0.49,1.71)$ \\
$\begin{array}{l}\text { Black (reference: non- } \\
\text { black) }\end{array}$ & $1.92(1.02,3.61)$ & $1.69(0.86,3.30)$ \\
$\begin{array}{l}\text { Type 1 diabetes } \\
\text { Type 2 diabetes }\end{array}$ & $0.61(0.19,2.02)$ & $0.30(0.07,1.33)$ \\
Hypoglycemic disorder & $1.29(0.63,2.65)$ & $0.77(0.29,2.10)$ \\
$\begin{array}{l}\text { Malnutrition/eating } \\
\text { disorders }\end{array}$ & $0.75(0.67,16.67)$ & $7.70(1.41,42.0)$ \\
$\begin{array}{l}\text { Congestive heart failure } \\
\text { End stage renal disease }\end{array}$ & $2.20(1.18,4.10)$ & $1.03(0.33,3.20)$ \\
Chronic liver disease/ & $0.60(0.21,1.72)$ & $0.45(0.89,4.11)$ \\
liver failure & $0.80(0.30,2.14)$ & $0.54(0.16,1.90)$ \\
HIV & $1.12(0.54,2.32)$ & $1.50(0.54,4.00)$ \\
Malignant neoplasm & $3.19(1.09,9.36)$ & $3.63(1.03,12.8)$ \\
$\begin{array}{l}\text { Insulin or insulin } \\
\text { secretagogue use }\end{array}$ & $1.32(0.70,2.48)$ & $1.31(0.55,3.12)$ \\
\hline
\end{tabular}

OR, odds ratios; $C I$, confidence interval; $B G$, blood glucose

OR and 95\% CI calculated for 209 CAV hypoglycemic episodes and 154 patient clusters

${ }^{a}$ OR for outcome of documented adjusted for all covariates shown in table

Table 3 shows the association between patient and hypoglycemic episode characteristics and the outcome of providerto-patient contact in unadjusted and fully adjusted models. On univariable analysis, increasing time from BG specimen collection to result, black race, CHF, and malignant neoplasm were significantly associated either positively or negatively with patient-to-provider contact. In the fully adjusted model, however, only time from specimen collection, hypoglycemic disorder, and malignant neoplasm were associated with the outcome. For each 1-hr increase in the time from BG collection to result, there was a $10 \%$ reduced odds of provider-topatient contact ( $95 \%$ CI, 2 to 17\%). The presence of a hypoglycemic disorder and malignancy were associated with higher likelihood of provider-to-patient contact, OR of 7.70 (95\% CI, 1.41, 42.0) and 3.63 (95\% CI, 1.03, 12.8), respectively.

Table 4 summarizes the documented clinical sequelae of the hypoglycemic CAV episodes. Overall, among the 128 episodes where the patient was contacted, no signs or symptoms were reported in $80.5 \%$. Among the 25 episodes where patient-reported symptoms were documented, the most commonly reported symptoms were weakness/fatigue (32.0\%); dizziness (16.0\%); tremor (12.0\%); non-specific symptoms (e.g. "felt hypoglycemic") (12.0\%); diaphoresis $(8.0 \%)$; confusion, altered mental status, or cognition $(8.0 \%)$; and slurred or altered speech $(8.0 \%)$.

A hypoglycemia-related serious adverse event was identified in 4 of the 128 (3.1\%) episodes where the patient was contacted (Table 4). Among these four episodes, EMS/ paramedic support was required in three cases, glucagon
Table 4 Clinical Sequelae of CAV for Hypoglycemia.

\begin{tabular}{ll}
\hline \hline & $\begin{array}{l}\text { No. of CAV hypoglycemic } \\
\text { episodes with documented } \\
\text { provider-to-patient contact }(\%)\end{array}$ \\
\hline $\begin{array}{l}\text { Documented hypoglycemic symptoms } \\
\text { No documented signs or }\end{array}$ & $103 / 128(80.5)$ \\
symptoms of hypoglycemia & \\
One or more hypoglycemic & $25 / 128(19.5)$ \\
signs or symptoms documented & $8 / 25(32.0)$ \\
Weakness/fatigue & $4 / 25(16.0)$ \\
Dizziness & $3 / 25(12.0)$ \\
Non-specific ("felt hypoglyce- & \\
mic") & $3 / 25(12.0)$ \\
Tremor & $2 / 25(8.0)$ \\
Diaphoresis & $2 / 25(8.0)$ \\
Confusion, altered mental & \\
status, or cognition & $2 / 25(8.0)$ \\
Slurred or altered speech & $1 / 25(4.0)$ \\
Hunger & $1 / 25(4.0)$ \\
Syncope & $1 / 25(4.0)$ \\
Feeling cold & $1 / 25(4.0)$ \\
Vision change & $124 / 128(96.9)$ \\
Documented hypoglycemia-related & $s e r i o u s$ adverse event \\
None identified & $4 / 128(3.1)$ \\
One or more serious adverse & \\
events documented & $3 / 4(75.0)$ \\
Need for EMS or paramedic & \\
support & \\
Hypoglycemia-related ED visit & $3 / 4(75.0)$ \\
within 24 hours of CAV & $2 / 4(50.0)$ \\
Need for glucagon & $1 / 4(25.0)$ \\
Loss of consciousness or & \\
syncope & $0 / 4(0.0)$ \\
Need for intravenous dextrose & $0 / 4(0.0)$ \\
Motor vehicle accident & $0 / 4(0.0)$ \\
Seizure & $0 / 4(0.0)$ \\
Cardiac ischemic event or & $0 / 4(0.0)$ \\
arrhythmia & \\
Coma or death & \\
\hline & \\
\hline
\end{tabular}

was required in two cases, and $\mathrm{ED}$ visit in 3 cases, and loss of consciousness/syncope occurred in 1 case. In case 1 , the patient had a CAV BG prior to being seen in outpatient clinic that day; during clinic, the patient remained symptomatic with severe lethargy and had a repeat fingerstick glucose of $28 \mathrm{mg} / \mathrm{dL}$, prompting treatment with IM glucagon and transfer to the ED. In case 2, the patient was contacted by the provider in response to a CAV BG and reported having experienced a syncopal event earlier in the day. The patient was asymptomatic at the time of communication with the provider after having self-treated with orange juice, and was advised to vigilantly monitor BG and go to the ED immediately if symptoms recurred. In case 3 , the provider contacted the patient in response to a CAV $\mathrm{BG}$ of $38 \mathrm{mg} / \mathrm{dL}$. At the time of communication, the patient was coherent, but reported shortness of breath. The provider advised the patient to eat and drink and go to the ED if dyspnea persisted. Approximately $2 \mathrm{~h}$ later, the patient presented to the ED and was found to be severely hypoglycemic and hypotensive, requiring hospital admission. In case 4 , the patient had a BG of $44 \mathrm{mg} / \mathrm{dL}$ occurring after an office visit with the ordering provider. There was no documentation of contact by the provider regarding the CAV BG. The patient was transported via EMS to the ED approximately $4 \mathrm{~h}$ later with moderate dyspnea, generalized 
malaise and weakness, had a BG of $33 \mathrm{mg} / \mathrm{dL}$, and was admitted. Notably, the patient had ESRD on dialysis and had missed a dialysis session.

\section{CONCLUSIONS}

To our knowledge, this study is the first to systematically examine provider responses to and patient outcomes of hypoglycemia triggering a CAV in the ambulatory setting. We found that (a) there was documented patient contact in response to CAVs less than two-thirds of the time, and those who were contacted had long delays; (b) a provider response to the CAV was rarely documented and the most common recommendation was to follow-up with their provider; and (c) there were low rates of documented serious adverse events in this population. Considering that this study only identified 213 CAVs for hypoglycemia over a 4-year period, this was not a common occurrence in our cohort. Nonetheless, our study exposed deficiencies in the current process of responding to CAV for hypoglycemia, which if designed to protect patients from harm, would need to be re-examined to ensure that there is a more consistent and prompt response.

We identified that the response to CAV for hypoglycemia in the ambulatory setting may be suboptimal for several reasons. First, nearly $40 \%$ of episodes had no documented evidence of provider-to-patient contact. Second, there was a substantial lag between the time of BG resulting to documented provider-to-patient contact. Although we cannot know for certain the exact interval of time between BG CAV resulting and provider-to-patient contact (as many providers may document this communication minutes to hours after the occurrence), we suspect that most providers would likely document this communication near the time that it occurred; thus, there is likely a clinically significant lag between the provider notification by the lab and the time that they contact the patient regarding the result. Considering that symptomatic hypoglycemia must be treated immediately, this draws into question the utility of provider-to-patient contact as an intervention. Finally, in $25 \%$ of episodes with provider-to-patient contact, the provider did not document any recommendations provided to the patient. It was surprising to us that the etiology of CAVs for hypoglycemia itself was not explicitly identified in the majority of cases, and it is not clear to what extent if any of this was due to a lack of exploration on the clinicians' part.

Despite the very low glucose threshold of the CAV in this study, documented patient symptoms and serious adverse outcomes were uncommon. In nearly $80 \%$ of episodes where there was provider-to-patient contact, no patient hypoglycemic signs or symptoms were documented. Since we do not know whether providers systematically inquired about hypoglycemic symptoms, we cannot definitively conclude that the prevalence of symptomatic hypoglycemia in this population is low. One or more serious adverse events was documented to occur in only 4 hypoglycemic episodes $(3.1 \%)$ where the patient was contacted, and none of these resulted in death. Another possibility for the low prevalence of documented hypoglycemic symptoms is impaired awareness of hypoglycemia (IAH), which is known to be a risk factor for severe hypoglycemia. ${ }^{12-15}$ In this retrospective study, it is difficult to know the extent to which this may have been a factor.

When there was documented patient contact, the prevalence of specific and actionable recommendations by the provider was relatively low. The most common documented action was reassigning responsibility by asking the patient to address this with another healthcare provider. We were surprised that only one episode had documentation about glucagon emergency kit availability. Although providers may have inquired about expired glucagon or patients may have already had glucagon at home, we suspect that the low rate of documentation about this reflects suboptimal adherence to the recommendation to ensure that glucagon is available as a rescue medication for all individuals at risk of $\mathrm{BG}<54 \mathrm{mg} / \mathrm{dL}{ }^{16}$

We found that the main predictors of provider-to-patient contact were time from $\mathrm{BG}$ collection to result, race, $\mathrm{CHF}$, and malignant neoplasm; however, in our fully adjusted model, the main predictors were time from BG collection to result, hypoglycemic disorder, and malignant neoplasm. The attenuation of the racial difference in adjusted models suggests that this difference may be due to the presence of other hypoglycemic risk factors. We hypothesize that a shorter interval between BG collection and laboratory resulting would be associated with a higher likelihood of provider-to-patient contact because providers might assume that patients would likely self-treat if persistently symptomatic hours from the specimen collection. We suspect that the higher odds of provider-patient contact in patients in patients with underlying malignancy is related to higher situational awareness in these patients, since severe hypoglycemia has been shown to be associated with cancer mortality. ${ }^{17}$ Similarly, there is likely greater degree of situational awareness in patients who are being actively followed for a serious hypoglycemic problem, such as insulinoma. We were surprised by the lack of association with T1DM (likely limited by small sample size) and/or use of insulin/insulin secretagogues, which clinically would be expected to confer the greatest risk of rapidly declining BG without intervention. ${ }^{18-20}$ Larger studies would be needed to better understand what clinical factors influence the outcome of patientprovider contact in response to hypoglycemic CAVs.

The main strengths of this study are the long study period (4 years), detailed ascertainment of patient histories through manual chart review, and inclusion of a broad number of clinical predictors. This study also has several limitations related to its retrospective design. The time between CAV result and patient contact was subject to variation given the difficulty in extracting the exact time that a patient was contacted from the EMR. Second, interpretation of provider and/ or patient response from review of the EMR is inherently subjective. It is also not possible to know if those patients who were not contacted experienced serious adverse events. 
Moreover, we were not able to determine whether provider level of training or specialty influenced the action or recommendations taken. Finally, these findings are generalizable to a high-resource academic medical center and further studies would be needed to understand the implications of a CAV hypoglycemia workflow in rural or less resourced settings.

In conclusion, this study found a low prevalence of CAVs for hypoglycemia for which the etiology remained largely unidentified, infrequent provider-to-patient contact, an inverse relationship between provider-patient contact and time of lab result, and a direct relationship with underlying malignancy and hypoglycemic disorders. Further prospective studies will be needed to definitively determine the prevalence of hypoglycemic symptoms and serious adverse events in relation to low ambulatory BG values to "calibrate" the CAV BG threshold, and evaluation of optimal provider response protocols.

Corresponding Author: Nestoras N. Mathioudakis, MD, MHS; Division of Endocrinology, Diabetes, \& Metabolism, Johns Hopkins University School of Medicine Baltimore, MD, USA (e-mail: nmathio1@jhmi.edu).

\section{Compliance with Ethical Standards:}

Conflict of Interest: Nestoras Mathioudakis was supported by grant K23DK111986-01 from the National Institute for Diabetes and Digestive and Kidney Diseases. The authors declared that there is no potential conflict of interest.

\section{REFERENCES}

1. Lundberg GD. Critical (panic) value notification: an established laboratory practice policy (parameter). JAMA 1990;263(5):709.

2. Seaquist ER, Anderson J, Childs B, et al. Hypoglycemia and diabetes: a report of a workgroup of the American Diabetes Association and the Endocrine Society. Diabetes Care 2013;36(5):1384-1395.

3. Group IHS. Glucose Concentrations of Less Than $3.0 \mathrm{mmol} / \mathrm{L}$ ( $54 \mathrm{mg} / \mathrm{dL}$ ) Should Be Reported in Clinical Trials: A Joint Position Statement of the American Diabetes Association and the European Association for the Study of Diabetes. Diabetes Care 2017;40(1):155-157.

4. Iqbal A, Heller S. Managing hypoglycaemia. Best Pract Res Clin Endocrinol Metab 2016;30(3):413-430.
5. Donnelly LA, Morris AD, Frier BM, et al. Frequency and predictors of hypoglycaemia in Type 1 and insulin-treated Type 2 diabetes: a population-based study. Diabet Med 2005;22(6):749-755.

6. Group UHS. Risk of hypoglycaemia in types 1 and 2 diabetes: effects of treatment modalities and their duration. Diabetologia 2007;50(6):11401147.

7. Mayo Clinic Laboratories Critical Values / Critical Results List. https:// www.mayocliniclabs.com/it-mmfiles/DLMP_Critical_Values_-_Critical Results_List.pdf. Accessed 20 March 2020.

8. MGH Clinical Laboratory Callback List. http://mghlabtest.partners.org/ CriticalValues.htm. Accessed 20 March 2020.

9. Abusamaan MS, Marzinke MA, Ashok A, et al. Hypoglycemic risk exposures in relation to low serum glucose values in ambulatory patients. Medicine (Baltimore) 2020;99(2):e18679.

10. Williams $\mathbf{R}$ 2000. A note on robust variance estimation for clustercorrelated data. Biometrics 56: 645-646. In.

11. Rogers W. 1993. sg17: Regression standard errors in clustered samples. Stata Technical Bulletin 13: 19-23.Reprinted in Stata Technical Bulletin Reprints, vol. 3, pp. 88-94. College Station, TX: Stata Press. In.

12. Geddes J, Schopman JE, Zammitt NN, Frier BM. Prevalence of impaired awareness of hypoglycaemia in adults with Type 1 diabetes. Diabet Med 2008;25(4):501-504.

13. Gold AE, MacLeod KM, Frier BM. Frequency of severe hypoglycemia in patients with type I diabetes with impaired awareness of hypoglycemia. Diabetes Care 1994;17(7):697-703.

14. Clarke WL, Cox DJ, Gonder-Frederick LA, Julian D, Schlundt D, Polonsky W. Reduced awareness of hypoglycemia in adults with IDDM. A prospective study of hypoglycemic frequency and associated symptoms. Diabetes Care 1995;18(4):517-522.

15. Olsen SE, Bjørgaas MR, Åsvold BO, et al. Impaired Awareness of Hypoglycemia in Adults With Type 1 Diabetes Is Not Associated With Autonomic Dysfunction or Peripheral Neuropathy. Diabetes Care 2016:39(3):426-433.

16. Association AD. 6. Glycemic Targets:. Diabetes Care 2020;43(Suppl 1):S66-S76.

17. Lee AK, Warren B, Lee CJ, et al. The Association of Severe Hypoglycemia With Incident Cardiovascular Events and Mortality in Adults With Type 2 Diabetes. Diabetes Care 2018;41(1):104-111.

18. Ratzki-Leewing A, Harris SB, Mequanint S, et al. Real-world crude incidence of hypoglycemia in adults with diabetes: Results of the InHypoDM Study, Canada. BMJ Open Diabetes Res Care 2018;6(1):e000503.

19. Cryer PE, Axelrod L, Grossman AB, et al. Evaluation and management of adult hypoglycemic disorders: an Endocrine Society Clinical Practice Guideline. J Clin Endocrinol Metab 2009;94(3):709-728.

20. Maloney A, Rosenstock J, Fonseca V. A Model-Based Meta-Analysis of 24 Antihyperglycemic Drugs for Type 2 Diabetes: Comparison of Treatment Effects at Therapeutic Doses. Clin Pharmacol Ther 2019;105(5):1213-1223.

Publisher's Note: Springer Nature remains neutral with regard to jurisdictional claims in published maps and institutional affiliations. 